\title{
EVALUACIÓN DEL COMPORTAMIENTO DE SOLUCIONES CON POTENCIALIDAD FUNGUICIDA MEDIANTE ENSAYOS DE BIODEGRADACIÓN EN LABORATORIO Y FT-IR
}

\author{
Murace, Mónica ${ }^{15}$; Saparrat, Mario ${ }^{15,16}$; Maly, Laura ${ }^{15}$ y Keil, Gabriel ${ }^{15}$
}

\section{RESUMEN}

En la República Argentina, el Arseniato de cobre cromatado (CCA-C) es una de las sustancias más utilizadas en el campo de la preservación industrial de las maderas. La toxicidad y fijación de dicho preservante aseguran para el material tratado una vida útil superior a los 30 años en condiciones de uso extremas.

El reemplazo del CCA es tendencia mundial debido a la presencia de arsénico (Ar) y cromo hexavalente $\left(\mathrm{Cr}^{+6}\right)$ en su composición. En este contexto, existe un creciente interés en el desarrollo de compuestos no tóxicos y con igual efectividad que el CCA.

El objetivo del trabajo fue evaluar el comportamiento de soluciones hidrosolubles de bajo compromiso ambiental en madera de Pinus ponderosa mediante su resistencia a la degradación fúngica y espectroscopía infrarroja transformada de Fourier (FT-IR). Se trabajó con albura de $P$. ponderosa Dougl. ex Laws. proveniente de ejemplares de una forestación de 22 años de edad ubicada en Neuquén (Argentina). Parte del material fue impregnado en autoclave con paraformaldehído (3 concentraciones); metaborato de sodio (2 concentraciones); fosfato-borato; preservante biodegradable en desarrollo y CCA-C (preservante de referencia).

En el material tratado fueron determinadas la penetración, absorción, retención nominal y real (IRAM 9600). Los ensayos de biodegradación se realizaron según IRAM 9518 con Gloeophyllum sepiarium (Wulf.: Fr.) P. Karst., hongo causante de pudrición castaña. Fueron utilizadas 360 probetas (40 por tratamiento/solución y concentración incluyendo al testigo). Del total, 180 fueron expuestas a situaciones de intemperismo simuladas en laboratorio a fin de determinar la permanencia de las soluciones de ensayo; el resto permitió determinar toxicidad. Toxicidad y permanencia fueron estimadas indirectamente, mediante la media de pérdida de peso (Pp \%) obtenida para cada tratamiento.

Estos datos permitieron determinar la clase de resistencia a la degradación fúngica y consecuentemente el comportamiento de las soluciones. Asimismo se procedió al análisis químico de las probetas de la totalidad de los tratamientos pertenecientes a ambos tipos de ensayo mediante FT-IR como tambien de aquellas tratadas sin exposición a la cepa fúngica (testigo de cada tratamiento).

Los espectros de absorción FT-IR fueron registrados entre los 4000 y $400 \mathrm{~cm}^{-1}$. Se seleccionaron bandas de referencia dentro de la región $800-1800 \mathrm{~cm}^{-1}$ que reflejan grupos funcionales característicos de los polímeros de la madera, y se estimaron sus intensidades relativas. El preservante biodegradable poseería el mayor potencial como sustituto del CCA. La toxicidad de dicha solución evidenciada mediante los ensayos de biodegradación (la madera resultó altamente resistente al igual que la tratada con CCA) y FT-IR como también mediante los parámetros de preservación obtenidos en el material, avalarían su aptitud. Por su parte, si bien manifestó tendencia al lixiviado, el material mantuvo su clase de resistencia/durabilidad.

Palabras clave: Preservantes alternativos, Toxicidad, Permanencia, FT-IR.

${ }_{15}$ Facultad de Ciencias Agrarias y Forestales, UNLP. La Plata, Buenos Aires, Argentina mmurace@gmail.com

16 INFIVE, UNLP-CCT La Plata CONICET 


\section{SUMMARY}

In the Argentine Republic, the chromated copper arsenate (CCA-C) is one of the most commonly used substances in the industrial wood preservation. The toxicity and fixing of this preservative ensure for the treated material a useful life more than 30 years in extreme conditions of use.

The replacement of the CCA is a world trend due to the presence of arsenic (Ar) and chrome hexavalent $\left(\mathrm{Cr}^{+6}\right)$ in its composition. In this context, there is a growing interest in the development of non toxic compounds and with equal effectiveness to the CCA.

The objective of the work was to evaluate the behavior of water-soluble solutions of low commitment environmental in wood of Pinus ponderosa through its resistance to fungal degradation and spectroscopy infrared transformed of Fourier (FT-IR). It worked with $P$. ponderosa Dougl. ex Laws. sapwood from 22 years old forestry located in Neuquén (Argentine). Part of the material was impregnated in autoclave with paraformaldehyde (3 concentration); metaborate of sodium (2 concentration); fosfato-borato; biodegradable preservative in development and CCA-C (preservative of reference).

In the treated material, penetration, absorption, nominal and real retention (IRAM 9600) were determined. The biodegradation tests were carried out according to IRAM 9518 with Gloeophyllum sepiarium (Wulf.: Fr.) P. Karst., a brown rot fungus. 360 specimens (40 by treatment / solution and concentration including control) were used. Of total, 180 were exposed to situations of weathering simulated in laboratory to determine the permanence of the solutions; the rest allowed to determine the toxicity. Toxicity and permanence were indirectly estimated, through the average weight loss obtained for each treatment.

These data allowed to determine the class of resistance to fungal degradation and consequently the behavior of the solutions. Also is proceeded to the chemical analysis of the specimens of all treatments belonging to both types of essay by FT-IR as well as those treated without exposition to the fungal strain (witness of each treatment).

The absorption spectra of FT-IR were registered between the 4000 and $400 \mathrm{~cm}-1$. Bands of reference within the region $800-1800 \mathrm{~cm}^{-1}$ that reflect functional groups characteristic of the wood polymers were selected, and their intensities relating were estimated. Biodegradable preservative would possess the greatest potential as substitute of the CCA. The toxicity of this solution, evidenced by the biodegradation tests (the wood was highly resistant as well as the CCA-treated) and FT-IR as also the preservation parameters obtained in material, would endorse their aptitude. Moreover, although tendency to leach, the material maintained its strength/durability class.

Key Words: Alternative preservatives, Toxicity, Permanence, FT-IR. 


\section{INTRODUCCIÓN}

Las prácticas de preservación permiten incrementar la vida útil de la madera en servicio como consecuencia de la resistencia al deterioro que le otorgan al material (Wottitz y Moreno, 2011). Los hongos xilófagos son los responsables del deterioro más frecuente y agresivo al que está expuesta la madera, producto de la ubicuidad de dichos organismos y su capacidad de colonización y degradación en periodos de tiempo relativamente cortos (Highley et al., 1994).

En la actualidad, el mercado maderero argentino se abastece principalmente de maderas exóticas. Las características químico-anatómicas de estas especies, su rápido crecimiento y cortos turnos de rotación determinan baja resistencia al deterioro fúngico (durabilidad), propiedad que condiciona su uso en situaciones propicias para que dicho proceso ocurra (Zabel y Morrell, 1992). En estas circunstancias, los procesos de preservación en particular, como así también el diseño y sistema constructivo permiten ampliar las situaciones de uso y, consecuentemente, las aplicaciones de las maderas actualmente disponibles (Encina, 2004). Las prácticas de preservación resultan estrategias eficaces de incremento de la durabilidad -durabilidad adquirida- en aquellas maderas poco resistentes (Díaz et al., 2003; Wottitz y Moreno, 2011).

En la República Argentina, el arseniato de cobre cromatado (CCA-C) es una de las sustancias impregnantes más utilizadas en el campo de la preservación industrial de las maderas (proceso bajo presión en autoclave) si bien otros principios activos y sus formulados se encuentran inscritos en el SENASA: Registro Nacional de Terapéutica Vegetal del Servicio Nacional de Sanidad y Calidad Agroalimentaria (IRAM 9600, 1998; Gherscovic, 2012). La elevada toxicidad del CCA hacia la mayoría de los agentes biológicos de deterioro y su fijación aseguran para la madera tratada una vida útil superior a los 30 años en una amplia variedad de ambientes (Coudert et al., 2014).

En el ámbito internacional, en los últimos 15 años hay una activa discusión en torno a la aplicación de CCA. Esto debido a los efectos nocivos que puede producir como también por los residuos originados por reemplazo del material preservado y de aquellos surgidos durante la preservación. En el país no existe una legislación que restrinja el uso de este principio activo, pese a ello la norma IRAM 9600 (1998) enumera restricciones para su aplicación. En este contexto el reemplazo del CCA en maderas orientadas a la construcción es una tendencia mundial (Rodríguez Barreal, 1998; Schmidt, 2006; Walker, 2006; Escobar, 2012). Por su parte, la Agencia de Protección Ambiental de Estados Unidos (EPA) determinó que su utilización no plantea un riesgo excesivo para el ser humano o el medio ambiente siempre que se tomen las medidas necesarias de mitigación de los mismos (Wottitz y Moreno, 2011).

La principal objeción respecto del uso de dicho preservante está relacionada con la presencia de arsénico $(\mathrm{Ar})$ y cromo hexavalente $\left(\mathrm{Cr}^{+6}\right)$ en su composición, ambos considerados como potenciales cancerígenos humanos (Walker, 2006). A causa de ello, en el año 2002 fue anunciada la decisión voluntaria por parte de la industria de la preservación de la madera de minimizar paulatinamente su aplicación; en los últimos años esta ha sido restringida en juegos infantiles, decks, senderos, cercos y demás usos residenciales.

Países como EEUU, Chile, Alemania y Suecia han optado por reemplazar el uso de este compuesto por productos alternativos como lo son el CCB (óxido de cromo, cobre y boro), el ACQ (compuesto cuaternario de cobre amoniacal) y el CBA (cobre azole); la EPA (2016) menciona además otros productos registrados para uso residencial como ser boratos, betaína polimérica, naftenato de cobre, entre otros, algunos de ellos aún no difundidos en Argentina (Borrallo Jiménez, 2007; Carmona y Barahona, 2010; Girardo, 2010; Moreno et al., 2010; Bozo et al., 2010; Wottitz y Moreno, 2011; Coudert et al., 2013). En este contexto existe un creciente interés en el desarrollo de compuestos no tóxicos, respetuosos con el medio ambiente y con igual efectividad que el CCA (Murgía, 2012). 


\section{OBJETIVO}

El objetivo del trabajo fue evaluar el comportamiento de soluciones hidrosolubles de bajo compromiso ambiental en madera de Pinus ponderosa mediante su resistencia a la degradación fúngica y espectroscopía infrarroja transformada de Fourier (FT-IR).

\section{MATERIALES Y MÉTODOS}

\section{Material de Ensayo}

Se trabajó con albura de Pinus ponderosa Dougl. ex Laws. (pino ponderosa) proveniente de ejemplares del primer raleo de una forestación de 22 años de edad ubicada en el sudoeste de la

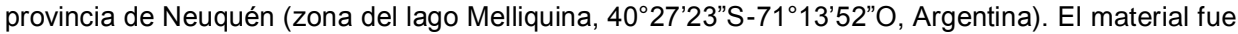
transformado en tablas de $20 \mathrm{~mm} \times 100 \mathrm{~mm} \times 400 \mathrm{~mm}$, posteriormente estivadas para su secado natural hasta constatar contenido de humedad en equilibrio higroscópico (HEH) mediante xilohigrómetro digital (Hydromette HT 85, de la marca GANN).

Logrado esto, parte de la madera fue impregnada en autoclave con las soluciones hidrosolubles ${ }^{17}$ : Paraformaldehido ${ }^{1}(\mathrm{P})$ en 3 concentraciones (P25\%, P50\% y $\mathrm{P} 100 \%$, respectivamente); metaborato de sodio ${ }^{2}$ (BO) en dos concentraciones (BO10\% y $\mathrm{BO} 15 \%$, de composición, en \%/p.s.: fosfato ácido de amonio, 10; sulfato de amonio, 60; ácido bórico, 20; borato de sodio, 10); fosfato-borato ${ }^{3}$ (FB $8 \%$, de composición, en g: fosfato monoamónico, 6.8; decaborato de sodio, 1.2; agua c.s.p., 100); arseniato de cobre cromatado (CCA-C) ${ }^{4}(2 \%$, de composición, en $\%$ : óxido de cromo, 45.63; óxido de cobre, 17.47; pentóxido de arsénico, 36.90) y preservante biodegradable en desarrollo ${ }^{5}$ (BIO $5 \%$, aún no patentado). En este estudio el CCA constituyó el preservante de referencia al momento de evaluar el comportamiento de aquellos considerados como potenciales sustitutos. Tratado el material se procedió a la determinación de los valores de penetración, absorción, retención nominal y de retención real (IRAM 9600, 1998).

Cumplida esta etapa, el material (tablas impregnadas y testigo sin preservar) fue transformado en listones de $20 \mathrm{~mm} \times 20 \mathrm{~mm} \times 400 \mathrm{~mm}$ a partir de los que se obtuvieron probetas cúbicas de $20 \mathrm{~mm}$ de lado, libres de defectos, a ser utilizadas en los ensayos de degradación acelerada según la normativa nacional vigente (IRAM 9518, 1962).

Se trabajó con 360 probetas (40 por tratamiento/solución y concentración). Del total, 180 fueron expuestas a situaciones de intemperismo simuladas en laboratorio a fin de determinar la permanencia de las soluciones de ensayo; el resto, permitió identificar la toxicidad de dichas soluciones.

\section{Ensayos de Biodegradación en Laboratorio}

\section{Material Fúngico}

Los ensayos de biodegradación fueron realizados con la cepa Gloeophyllum sepiarium (Wulf.: Fr.) P. Karst. cepa 735 (LPSC) - Cepario del Instituto Spegazzini, La Plata - causante de pudrición castaña, especie frecuentemente asociada al deterioro de la madera en servicio, particularmente de coníferas (Wright y Deschamps, 1976/77; Schmidt, 2006). Obtenido el cultivo, fue repicado a cajas de Petri con medio Agar Extracto de Malta (MEA 2\%) de constitución: peptona, $1 \mathrm{~g}$; extracto de malta, $20 \mathrm{~g}$; glucosa, $20 \mathrm{~g}$; agar, $20 \mathrm{~g}$; agua destilada, $1000 \mathrm{~cm}^{3}$. Posteriormente las cajas fueron colocadas en estufa a $25^{\circ} \mathrm{C}$ por 10 días a los efectos de obtener el inóculo necesario para el establecimiento de los ensayos.

\footnotetext{
$17^{*} 1$ Formulado en el Centro de Investigación y Desarrollo en Tecnología de Pinturas (CIDEPINT); ${ }^{2}$ Formulado en CIDEPINT; ${ }^{3}$ Formulado por Empresa Química Bosques; ${ }^{4}$ Formulado en INTI Madera \& Muebles; ${ }^{5}$ Formulado por Dr. Murgía UNL - CONICET.
} 


\section{- Instalación de los Ensayos - Procedimiento}

Los ensayos fueron realizados aplicando la técnica del soil block (IRAM 9518). En frascos de vidrio de $500 \mathrm{~cm}^{3}$ de capacidad fueron colocados $43 \mathrm{~cm}^{3}$ de agua destilada y $200 \mathrm{~g}$ de una mezcla de tierra $(40 \mathrm{~g})$ y arena $(160 \mathrm{~g})$ previamente secada en estufa a $103^{\circ} \mathrm{C} \pm 2$ durante $72 \mathrm{~h}$ y' posteriormente tamizada. Llenados los frascos, sobre el sustrato fueron apoyadas dos tablillas (feeder strips) de madera blanda (Salix spp) de $20 \mathrm{~mm}$ de lado y $4 \mathrm{~mm}$ de espesor. Posteriormente, cada frasco fue tapado con algodón y esterilizado en autoclave durante 30 minutos a 1.5 atm de presión. Cumplida esta etapa se procedió a la inoculación, la cual fue realizada mediante trozos de micelio fúngico de $10 \mathrm{~mm}$ de diámetro colocados sobre cada feeder strips. Una vez que el micelio cubrió las tablillas (25 días aproximadamente) sobre cada una de ellas fue colocada una probeta de ensayo (dos por frasco) previa determinación de su peso inicial en equilibrio higroscópico ( $\mathrm{Pi}$ ) y esterilización en autoclave a $1 / 2$ atm de presión durante 20 minutos. De este modo quedaron instalados los ensayos que permitieron determinar la toxicidad de las soluciones preservantes como también el ensayo testigo.

En cuanto a las probetas pertenecientes a los ensayos de permanencia, antes de su incorporación en los frascos de pudrición (preparados del modo indicado) fueron pesadas (Pi) y luego expuestas a lavado por inmersión en agua destilada $(2 \mathrm{~h})$, a estacionamiento en laboratorio a temperatura ambiente $\left(25 \pm 2{ }^{\circ} \mathrm{C}\right.$ durante $\left.16 \mathrm{~h}\right)$ y posterior calentamiento en estufa $\left(50 \pm 2{ }^{\circ} \mathrm{C}\right.$ por 6 h), secuencia repetida 10 veces. Cumplida dicha simulación de intemperismo (IRAM 9518) fueron esterilizadas del modo descrito e incorporadas en los frascos de pudrición sobre cada feeder streps colonizado por la cepa xilófaga.

La totalidad del material (40 probetas por tratamiento, 20 por tipo de ensayo) estuvo expuesto a deterioro durante 90 días a $27 \pm 2^{\circ} \mathrm{C}$ y $70 \%$ de HR. Transcurrido este tiempo, las probetas fueron extraídas de los frascos y acondicionadas en laboratorio (30 días aproximadamente) bajo condiciones controladas de temperatura y humedad $\left(25 \pm 2^{\circ} \mathrm{C}\right.$ y $70 \%$ de $\mathrm{HR}$ ) hasta comprobar peso en equilibrio higroscópico (Pf). Con los valores de Pi y Pf obtenidos fue calculada la media de pérdida de peso ( $\mathrm{Pp} \%$ ) para cada condición de la madera (tratamiento) y tipo de determinación (toxicidad, permanencia) empleando la fórmula:

$$
\operatorname{Pp}(\%)=\frac{P i(g)-P f(g)}{P i(g)} \times 100
$$

Calculada la $\mathrm{Pp}(\%)$, los datos obtenidos permitieron determinar la resistencia de la madera testigo e impregnada a la degradación fúngica (durabilidad natural y adquirida, respectivamente) según lo indica la escala de Findlay (Norma ASTM D-2017 81/1986): Altamente resistente (AR): 0\%-10\% Pp; Resistente (R): 11\%-24\% Pp; Moderadamente resistente (MR): $25 \%$ $44 \% \mathrm{Pp}$; No resistente (NR): >45\% Pp.

La durabilidad o resistencia a la degradación (definida en base al porcentaje medio de Pp obtenido) adquirida por el material no expuesto a intemperismo constituyó un indicador indirecto de la toxicidad de los preservantes sobre G. sepiarium. Por su parte, la durabilidad adquirida por la madera determinada mediante ensayos de permanencia permitió evaluar la capacidad de fijación de los preservantes.

El comportamiento de los impregnantes al lixiviado - permanencia - fue evaluado por comparación de porcentajes medios de pérdidas de peso ( $\mathrm{Pp} \%$ ): $\mathrm{Pp}(\%)$ del material sin exposición a intemperismo (toxicidad) vs Pp (\%) del material expuesto a intemperismo. El conocimiento de dichos atributos es de importancia teniendo en cuenta la alta toxicidad y fijación del CCA. La durabilidad de la madera actuaría como indicador de la potencialidad que poseen dichas soluciones en transformarse en posibles alternativas al uso del CCA en cuanto a la protección frente al ataque por hongos xilófagos. 


\section{Espectroscopía Infrarroja Transformada de Fourier (FT-IR)}

El comportamiento de las soluciones (toxicidad y permanencia) fue evaluado también mediante la degradación de los polímeros de la madera por G. sepiarium, por lo cual se procedió al análisis químico de las probetas de la totalidad de los tratamientos (madera preservada y sin tratar) pertenecientes a ambos tipos de ensayo. Material tratado y testigo ( $\mathrm{T}$ ) sin exposición a la degradación fúngica tambien fue analizado y considerado como testigo (t).

Material particulado correspondiente a la superficie de cada una de las muestras fue obtenido mediante lima metálica $(<0,4 \mathrm{~mm})$, luego triturado con nitrógeno líquido en un mortero de porcelana y homogeneizado el tamaño de sus partículas utilizando un lienzo de voile.

Con el fin de eliminar los extraíbles hidro y liposolubles de cada muestra obtenida, estas fueron expuestas a una serie de lavados secuenciales utilizando agua caliente, Triton $X-100$, etanol, metanol y acetona siguiendo el procedimiento reportado por Cvikrova et al. (2006) y Espiñeira et al. (2011). El material resultante fue secado a $80{ }^{\circ} \mathrm{C}$ y mantenido en una atmósfera libre de humedad hasta el momento de proceder al armado de las pastillas para FT-IR.

Para obtener los espectros FT-IR fueron elaboradas pastillas de $13 \mathrm{~mm}$ de diámetro con $2 \mathrm{mg}$ de cada muestra seca y $20 \mathrm{mg}$ de bromuro de potasio grado infrarrojo así como también pastillas blanco con solo $22 \mathrm{mg}$ de bromuro de potasio. Los espectros de absorción FT-IR fueron registrados entre 4000 y $400 \mathrm{~cm}^{-1}$ empleando un espectrómetro modelo Spectrum (Perkin- ElmerInstruments).

El análisis espectral se realizó a una velocidad de $1 \mathrm{~cm} \mathrm{~s}^{-1}$ con $4 \mathrm{~cm}^{-1}$ de resolución bajo atmósfera de aire seco (cámara con silicagel), siendo obtenido un espectro promedio resultado de 64 escaneos.

Las mediciones se realizaron dos veces por muestra analizada. Para el procesamiento de los datos se utilizó el software EZ-OMNIC y se llevó a cabo la corrección de cada espectro sustrayendo automáticamente aquel correspondiente a la pastilla blanco obtenido inmediatamente antes del registro de cada muestra.

Se seleccionaron bandas de referencia dentro de la región $800-1800 \mathrm{~cm}^{-1}$ (Cuadro $\mathrm{N}^{\circ} 1$ ), que reflejan grupos funcionales característicos de los polímeros de la madera, y se estimaron sus intensidades relativas siguiendo a Luna et al. (2015) y a Pandey y Pitman (2003). De acuerdo con estos últimos autores, diferencias $\leq$ a 0,10 entre valores de absorbancia relativa no se consideraron como tal.

\section{Cuadro $\mathrm{N}^{\circ} 1$ \\ ASIGNACION DE BANDAS DE ABSORCION EN LOS ESPECTROS FT-IR DE LAS MUESTRAS DE MADERA ANALIZADAS}

\begin{tabular}{|c|l|l|}
\hline $\begin{array}{c}\text { Número de } \\
\text { Onda } \\
\left(\mathbf{c m} \mathbf{-}^{-1}\right)\end{array}$ & \multicolumn{1}{|c|}{ Grupos Funcionales } & \multicolumn{1}{|c|}{ Referencia } \\
\hline 896 & Vibraciones de los grupos C1-H en celulosa. & (Hinterstoisser et al., 2001) \\
\hline 1164 & Vibraciones de los grupos glicosídicos C-O-C. & (Saparrat et al., 2010) \\
\hline 1508 & Vibraciones de los esqueletos aromáticos. & (Saparrat et al., 2010) \\
\hline 1734 & $\begin{array}{l}\text { Grupos carbonilos no conjugados de pectinas y y } \\
\text { hemicelulosas. }\end{array}$ & (Luna et al., 2015) \\
\hline
\end{tabular}




\section{- $\quad$ Análisis Estadístico}

Determinados los porcentajes medios de pérdida de peso (Pp\%) -variable respuesta para pudrición castaña en los ensayos de toxicidad y permanencia- fue realizado un análisis descriptivo a fin de obtener estadísticos básicos para cada tratamiento.

Con el propósito de comparar el efecto de las distintas soluciones impregnantes sobre la cepa xilófaga de ensayo fueron realizados ANOVA.

Al comprobar que los datos no cumplieron con los supuestos del análisis de la varianza, fue realizado un análisis de la varianza no paramétrico, prueba de Kruskal- Wallis (KW); posteriormente se aplicó la prueba de Mann Whitney para comparar los valores de Pp (\%) entre tipo de ensayo (toxicidad vs permanencia).

Para el análisis descriptivo e inferencial fue utilizado el software Infostat.

\section{RESULTADOS - DISCUSIÓN}

\section{Valores de los Parámetros de Impregnación Obtenidos Mediante Vacío - Presión}

Cuadro $\mathrm{N}^{\circ} 2$.

Los valores de penetración, absorción, retención nominal y real son detallados en el

Cuadro $\mathrm{N}^{\circ} 2$

PARÁMETROS DE IMPREGNACIÓN POR PRODUCTO Y CONCENTRACIÓN

\begin{tabular}{|c|c|c|c|c|}
\hline Tratamientos ${ }^{*}$ & $\begin{array}{l}\text { Absorción } \\
\left(\mathrm{kg} \cdot \mathrm{m}^{-3}\right)\end{array}$ & $\begin{array}{l}\text { Retención nominal } \\
\left(\mathrm{kg} \cdot \mathrm{m}^{-3}\right)\end{array}$ & $\begin{array}{l}\text { Retención real } \\
\left(\mathrm{kg} \cdot \mathrm{m}^{-3}\right)\end{array}$ & $\begin{array}{c}\text { Penetración } \\
(\%)\end{array}$ \\
\hline CCA-C & 636,871 & 8,789 & 7,224 & \\
\hline P100 & 636,101 & 636,101 & 522,875 & \\
\hline P50 & 504,539 & 252,269 & 207,366 & Total \\
\hline P25 & 608,030 & 234,702 & 192,925 & 100 \\
\hline BO15 & 499,079 & 74,862 & 61,536 & \\
\hline $\mathrm{BO} 10$ & 448,006 & 44,801 & 36,826 & \\
\hline FB & 551,356 & 44,108 & 36,257 & \\
\hline $\mathrm{BIO}$ & 542,022 & 27,101 & 22,277 & \\
\hline
\end{tabular}

* CCA-C: Arseniato de cobre cromatado al 2\%; P: Paraformaldehído al $100 \%, 50 \%$ y $25 \%$; BO:

Metaborato de sodio al 15\% y 10\%; FB: Fosfato-borato al 8\%; BIO: Preservante biodegradable al 5\%.

Según se indica en el Cuadro $N^{\circ} 2$, la penetración de las distintas soluciones fue total (madera impregnable) en correspondencia con el atributo albura penetrable que poseen distintas especies de pinos. Asimismo se correspondió con lo sugerido para los productos de madera impregnados a ser usados en distintas categorías de riesgo.

En cuanto al CCA, el valor medio de retención nominal también fue el esperado (IRAM 9600); para el resto de los productos ensayados no se registran antecedentes.

Lo expuesto evidencia la efectividad del tratamiento en cuanto al método elegido y al tiempo de vacío y de presión considerado. 


\section{Durabilidad Adquirida por la Albura de Pino Ponderosa Estimada Mediante Ensayos de Toxicidad}

En el Cuadro $\mathrm{N}^{\circ} 3$ son presentados los valores hallados a partir del análisis descriptivo y mediante la prueba KW para la variable pérdida de peso (Pp) por tratamiento producto de la exposición de la albura de pino ponderosa a la cepa $G$. sepiarium.

\section{Cuadro $\mathrm{N}^{\circ} 3$ \\ VALORES MEDIOS Y MEDIANAS DE PÉRDIDA DE PESO Y CLASE DE RESISTENCIA POR TRATAMIENTO OBTENIDOS PARA LA MADERA EXPUESTA A G. sepiarium}

\begin{tabular}{|c|c|c|c|c|c|c|c|c|c|}
\hline \multirow{2}{*}{ Estadístico } & \multicolumn{9}{|c|}{ Tratamientos* } \\
\hline & CCA-C & P100 & P50 & P25 & B015 & B010 & FB & BIO & $\mathbf{T}$ \\
\hline $\begin{array}{l}\text { Media (\%) } \\
(\mathrm{CV}) \\
\text { Resistencia** }\end{array}$ & $\begin{array}{r}5,39 \\
(23,48) \\
\text { AR }\end{array}$ & $\begin{array}{r}36,66 \\
(30,7) \\
\text { MR }\end{array}$ & $\begin{array}{r}30,34 \\
(28,71) \\
M R\end{array}$ & $\begin{array}{r}27,32 \\
(34,10) \\
M R\end{array}$ & $\begin{array}{r}14,07 \\
(36,83) \\
R\end{array}$ & $\begin{array}{r}5,62 \\
(33,48) \\
\text { AR }\end{array}$ & $\begin{array}{r}11,66 \\
(28,32) \\
R\end{array}$ & $\begin{array}{r}3,72 \\
(54,25) \\
\text { AR }\end{array}$ & $\begin{array}{r}43,72 \\
(21,39) \\
M R\end{array}$ \\
\hline $\begin{array}{l}\text { Mediana (\%) } \\
\text { Prueba KW }\end{array}$ & 5,47 & 35,39 & 30,67 & $\begin{array}{r}30,36 \\
25,94 \mathrm{P}_{<}\end{array}$ & $\begin{array}{r}13,28 \\
0001\end{array}$ & 5,08 & 11,49 & 3,75 & 46,71 \\
\hline
\end{tabular}

De acuerdo con lo esperado, el mayor porcentaje medio de pérdida de peso ( $\mathrm{Pp}$ ) fue obtenido para el material testigo (T). La baja resistencia a la degradación de la albura de pino ponderosa expuesta a $G$. sepiarium se corresponde con los antecedentes registrados a nivel mundial y en el país para esta y otras especies de coníferas respecto de su alta susceptibilidad a este tipo de deterioro (Bernardis y Popoff, 2009; USDA Forest Service, 2010; Murace et al., 2014).

Por su parte, los porcentajes de Pp obtenidos determinaron clases de resistencia diferentes entre grupos de tratamientos. En este sentido, la madera tratada con las 3 concentraciones de $\mathrm{P}$ resultó Moderadamente Resistente (MR) en coincidencia con lo obtenido para el T; aquella con FB y BO15 se comportó como Resistente (R); en cuanto al BO10 y el BIO determinaron que la madera se comporte como Altamente Resistente (AR) al igual que la tratada con CCA.

Para las muestras impregnadas con la solución BO10, opuestamente a lo esperado, a menor concentración y valor de retención (Cuadro №2) fue obtenida mayor durabilidad (Cuadro $\mathrm{N} \times 3)$.

La existencia de diferencias significativas entre las medianas de Pp de los tratamientos (prueba de $\mathrm{KW} \mathrm{H}=125,94$ y su $\mathrm{p}$-value $<0,0001$ ) indicó que los productos ensayados influyeron sobre la Pp y de forma diferencial en correspondencia con los tipos de durabilidad obtenidos y, a causa de ello, se corrobora la diferente toxicidad de los preservantes, definiendo distintos grupos mediante comparación de rangos (Cuadro $\mathrm{N}^{\circ} 4$ ).

Lo expuesto determinó que en esta instancia BIO y BO10 sean considerados como potenciales sustitutos del CCA. 


\section{Cuadro $\mathrm{N}^{\circ} 4$ \\ COMPARACIÓN DE RANGOS ENTRE TRATAMIENTOS PERTENECIENTES A LOS ENSAYOS DE TOXICIDAD}

\begin{tabular}{|c|r|r|r|r|c|}
\hline Tratamientos* $^{*}$ & \multicolumn{1}{|l|}{ Ranks $^{\star *}$} & \multicolumn{1}{|l|}{} \\
\hline BIO & 14,25 & A & & & \\
\hline BO10 & 30,83 & A & & & \\
\hline CCA-C & 30,95 & A & & & \\
\hline FB & 61,87 & & B & & \\
\hline BO15 & 71,94 & & B & & \\
\hline P25 & 101,88 & & & C & \\
\hline P50 & 107,11 & & & C & D \\
\hline P100 & 118,65 & & & C & D \\
\hline T & 132,31 & & & & D \\
\hline
\end{tabular}

* $\mathrm{BIO}$ : Preservante biodegradable al 5\%;

BO10: Metaborato de sodio al 10\%;

CCA-C: Arseniato de cobre cromatado al $2 \%$;

FB: Fosfato-borato al 8\%; BO15: Metaborato de sodio al $15 \%$;

P: Paraformaldehído al $25 \%, 50 \%$ y $100 \%$;

T: testigo sin impregnar.

**Letras distintas indican diferencias significativas $(p<=0,05)$.

\section{Durabilidad Adquirida por la Albura de Pino Ponderosa Estimada Mediante Ensayos de Permanencia}

En el Cuadro $\mathrm{N}^{\circ} 5$ son presentados los valores hallados a partir del análisis descriptivo y mediante la prueba KW para la variable porcentaje de pérdida de peso ( $\mathrm{Pp}$ ) por tratamiento producto de la exposición de la albura de Pino ponderosa a ciclos de intemperismo y posteriormente a la acción de la cepa $G$. sepiarium.

\section{CUADRO $N^{\circ} 5$}

VALORES MEDIOS Y MEDIANAS DE PÉRDIDA DE PESO Y CLASE DE RESISTENCIA POR TRATAMIENTO OBTENIDOS MEDIANTE ENSAYOS DE PERMANENCIA PARA LA MADERA EXPUESTA A G. sepiarium

\begin{tabular}{|c|c|c|c|c|c|c|c|c|c|}
\hline \multirow{2}{*}{ Estadístico } & \multicolumn{9}{|c|}{ Tratamientos ${ }^{\star}$} \\
\hline & CCA-C & P100 & P50 & P25 & B015 & B010 & FB & $\mathrm{BIO}$ & $\mathbf{T}$ \\
\hline $\begin{array}{l}\text { Media (\%) } \\
(\mathrm{CV}) \\
\text { Resistencia** }\end{array}$ & $\begin{array}{r}6,97 \\
(61,84) \\
\text { AR }\end{array}$ & $\begin{array}{r}42,31 \\
(29,85) \\
M R\end{array}$ & $\begin{array}{r}33,83 \\
(28,26) \\
M R\end{array}$ & $\begin{array}{r}28,99 \\
(29,88) \\
M R\end{array}$ & $\begin{array}{r}21,32 \\
(41,06) \\
R\end{array}$ & $\begin{array}{r}14,32 \\
(18,77) \\
R\end{array}$ & $\begin{array}{r}17,27 \\
(37,63) \\
R\end{array}$ & $\begin{array}{r}9,15 \\
(47,01) \\
\text { AR }\end{array}$ & $\begin{array}{r}27,90 \\
(20,03) \\
M R\end{array}$ \\
\hline $\begin{array}{l}\text { Mediana (\%) } \\
\text { Prueba KW }\end{array}$ & 4,37 & 39,46 & 32,20 & $\begin{array}{r}31,69 \\
01,94 ; P\end{array}$ & $\begin{array}{r}21,24 \\
0,0001\end{array}$ & 14,38 & 18,86 & 8,42 & 28,10 \\
\hline
\end{tabular}

${ }^{*}$ CCA-C: Arseniato de cobre cromatado al $2 \%$;

P: Paraformaldehído al $100 \%, 50 \%$ y $25 \%$;

BO15, BO10: Metaborato de sodio al $15 \%$ y $10 \%$;

FB: Fosfato-borato al 8\%; BIO: Preservante biodegradable al 5\%;

T: testigo sin impregnar.

** Clase de resistencia: AR, altamente resistente: 0\%-10\% Pp; R, resistente: $11 \%-24 \% \mathrm{Pp}$; MR, moderadamente resistente: $25 \%-44 \% \mathrm{Pp}$; NR, no resistente: $>45 \% \mathrm{Pp}$.

En coincidencia con lo hallado mediante ensayos de toxicidad (Cuadro $\mathrm{N}^{\circ} 3$ ), la albura de pino ponderosa sin tratamiento $(\mathrm{T})$ se comportó como MR. 
En esta instancia, el menor porcentaje medio de $\mathrm{Pp}$ obtenido (Cuadro $\mathrm{N}^{\circ} 3$ vs Cuadro $N^{\circ} 5$ ) podría deberse a la pérdida de azucares libres en la madera consecuencia de los ciclos de intemperismo, carbohidratos esenciales para el inicio del proceso de colonización y degradacion fúngica (Schwarze, 2007).

Respecto del material preservado, si bien en la totalidad de los tratamientos se determinó un aumento de $\mathrm{Pp}$ (indicando indirectamente pérdida de las soluciones por lavado, Cuadro $\mathrm{N}^{\circ} 3$ vs Cuadro $\mathrm{N}^{\circ}$ ), solo para el material preservado con $\mathrm{BO} 10$ dicho incremento significó un cambio en su rango de durabilidad, de AR paso a comportarse como $\mathrm{R}$.

Lo hallado reflejó la mayor lixiviación de esta solución en relación al resto de los preservantes (tal la experiencia con bórax - ácido bórico) como también determinó para ella un comportamiento similar al del resto de las formulaciones con boro en su composición (BO15 y FB) en cuanto a la durabilidad otorgada al material (Cuadro $\mathrm{N}^{\circ} 5$ ).

En cuanto a la madera con $\mathrm{P}$, mantuvo su condición de MR y, consecuentemente, comportamiento similar al T; de igual modo la impregnada con la solución BO15 y la tratada con FB, ambas conservaron su comportamiento $\mathrm{R}$ y aquellas con CCA y BIO mantuvieron su condición de $A R$ (Cuadro $\mathrm{N}^{\circ} 3$ vs Cuadro $\mathrm{N}^{\circ} 5$ ).

La prueba de KW, nuevamente determinó que las diferencias entre las medianas de Pp de los tratamientos fueron significativas $(H=125,94$ y su $\mathrm{p}$-value $<0,0001)$ corroborando el comportamiento diferencial de las soluciones en cuanto a permanencia y durabilidad otorgada al material, tendencia que se vio reflejada mediante comparación de rangos (Cuadro $\mathrm{N}^{\circ} 6$ ).

De acuerdo con esto, la solución identificada como BIO continúa resultando la más competente en el marco de la búsqueda de preservantes potencialmente sustitutos del CCA.

\section{Cuadro $\mathrm{N}^{\circ} 6$}

COMPARACIÓN DE RANGOS ENTRE TRATAMIENTOS PERTENECIENTES A LOS ENSAYOS DE PERMANENCIA

\begin{tabular}{|c|r|r|r|r|r|}
\hline Tratamientos $^{*}$ & \multicolumn{1}{|c|}{ Ranks $^{\star *}$} & \multicolumn{1}{|l|}{} \\
\hline CCA-C & 15,00 & A & & & \\
\hline BIO & 21,56 & A & & & \\
\hline BO10 & 42,40 & A & B & & \\
\hline FB & 51,38 & & B & & \\
\hline BO15 & 66,76 & & B & C & \\
\hline T & 90,05 & & & C & D \\
\hline P25 & 95,38 & & & & D E \\
\hline P50 & 106,39 & & & & D E \\
\hline P100 & 122,55 & & & & E \\
\hline
\end{tabular}

* CCA-C: Arseniato de cobre cromatado al 2\%;

BIO: preservante biodegradable al $5 \%$;

BO10: Metaborato de sodio al $10 \%$ :

FB: Fosfato-borato al 8\%; BO15: Metaborato de sodio al 15\%;

T: testigo sin impregnar;

P: Paraformaldehído al $25 \%, 50 \%$ y $100 \%$.

${ }^{* *}$ Letras distintas indican diferencias significativas $(p<=0,05)$.

La prueba de Mann Whitney (Cuadro $N^{\circ} 7$ ) demostró la existencia de diferencias significativas entre ensayos para las soluciones en base a Boro y $\mathrm{BIO}$ y, consecuentemente, su mayor lixiviación respecto del resto de las soluciones. 
Cuadro $\mathrm{N}^{\circ} 7$

PRUEBA DE MW: COMPARACIÓN DE LAS MEDIANAS DE PÉRDIDA DE PESO ENTRE TIPO DE ENSAYO POR TRATAMIENTO PARA LA ALBURA DE P. PONDEROSA EXPUESTA A G. sepiarium

\begin{tabular}{|c|c|c|c|c|c|c|c|c|c|c|}
\hline Tratam $^{*}$ & Clasif. & Variable & Grupo & Grupo & $N(1)$ & $n(2)$ & Mediana(1) & Mediana(2) & W & $p(2)$ \\
\hline CCA-C & Tx-Pr & $\mathrm{Pp} \%$ & $i$ & 2 & 20 & 10 & 5,47 & 4,37 & 146,50 & 0,7084 \\
\hline P100 & Tx-Pr & $\mathrm{Pp} \%$ & 1 & 2 & 17 & 19 & 35,39 & 39,46 & 284,00 & 0,3337 \\
\hline P50 & $\mathrm{Tx}-\operatorname{Pr}$ & $\mathrm{Pp} \%$ & 1 & 2 & 19 & 18 & 30,67 & 32,20 & 373,50 & 0,3384 \\
\hline P25 & $T x-P r$ & $\mathrm{Pp} \%$ & 1 & 2 & 17 & 17 & 30,36 & 31,69 & 315,00 & 0,5467 \\
\hline $\mathrm{BO} 15$ & $T x-\operatorname{Pr}$ & $\mathrm{Pp} \%$ & 1 & 2 & 16 & 17 & 13,28 & 21,24 & 211,00 & 0,028 \\
\hline $\mathrm{BO} 10$ & $T x-P r$ & $\mathrm{Pp} \%$ & 1 & 2 & 18 & 10 & 5,08 & 14,38 & 232,50 & $<0,0001$ \\
\hline FB & Tx-Pr & $\mathrm{Pp} \%$ & 1 & 2 & 15 & 16 & 11,49 & 18,86 & 179,00 & 0,0159 \\
\hline $\mathrm{BIO}$ & Tx-Pr & $\mathrm{Pp} \%$ & 1 & 2 & 12 & 18 & 3,75 & 8,42 & 98,00 & 0,0002 \\
\hline$T$ & $T x-\operatorname{Pr}$ & $\mathrm{Pp} \%$ & 1 & 2 & 16 & 20 & 46,71 & 28,10 & 427,00 & $<0,0001$ \\
\hline
\end{tabular}

* CCA-C: Arseniato de cobre cromatado al $2 \%$;

P: Paraformaldehído al $100 \%, 50 \%$ y $25 \%$;

BO15, BO10: Metaborato de sodio al $15 \%$ y $10 \%$;

FB: Fosfato-borato al $8 \%$;

BIO: preservante biodegradable al $5 \%$;

T: testigo sin impregnar.

En referencia a los boratos, lo obtenido es compatible con los antecedentes registrados en torno a la tendencia al lixiviado de estos preservantes. A causa de ello, distintas investigaciones fueron conducidas a fin de lograr su fijación en la madera mediante la formación de complejos boro - taninos flavonoides; sílice-boro; de soluciones hidrosolubles de ácido bórico, gelatina y taninos; de complejos taninos - hexaminas - ácido bórico, entre otros (Walker, 2006; Caldeira, 2010; Thévenon et al., 2010; Carmona et al., 2012).

En cuanto a $\mathrm{BIO}$, pese al lixiviado continúa siendo el más competente en la búsqueda de compuestos alternativos, particularmente por su toxicidad como también por los parámetros de impregnación obtenidos. Al igual que el CCA, BIO determinó que la madera se comporte como altamente resistente a menor concentración y retenciones respecto del resto de las soluciones y absorción del orden de los $500 \mathrm{~kg}$ de producto tóxico por metro cúbico de albura tratada. Asimismo el material expuesto a intemperismo mantuvo su clase de resistencia.

La efectividad de un tratamiento es determinada por la profundidad de penetración del preservante, por la distribución de dicha sustancia, por la cantidad de producto retenido como tambien por su toxicidad a los que se suma la permanencia como rasgo determinante de la situación de uso del material tratado (Paes et al., 2008; Lebow, 2010). Murguía (2012) aporta antecedentes respecto de las ventajas de esta solución.

Por su parte, para el material tratado con CCA, la falta de diferencias significativas entre ensayos para las medianas de $\mathrm{Pp}$ y la elevada clase de resistencia estimada mediante los ensayos establecidos (Cuadros $\mathrm{N}^{\circ} 3$ a $\mathrm{N}^{\circ} 6$ ) se corresponden con los antecedentes en torno a la alta toxicidad y permanencia de este producto. Existen antecedentes generados en laboratorio acerca de la resistencia de Pinus sylvestris impregnado con CCA (Pp inferior al 10\%) a la degradación por G. sepiarium consecuencia de la condición de cepa no tolerante al cobre (Kose y Kartal, 2010).

Respecto del paraformaldehído, los resultados obtenidos en ambos tipos de ensayo no avalan su utilización ante la necesidad de incrementar la durabilidad de la madera. Esta resina fenólica, resultante de la condensación del fenol/fenoles con un aldehído -en este caso Paraformaldehído-, conocida también como resina fenol-formol o fenol-formaldehído, está indicada ante requerimientos de aislamiento térmico y eléctrico por lo que encuentra aplicaciones en campos que van desde el aeroespacial al electrónico ya sea como polvos de moldeo, resinas para 
laminados, adhesivos, impregnantes y recubrimientos superficiales, entre otros (Martín Moreno, 2005, tomado de Keil et al., 2012).

\section{Espectroscopía Infrarroja Transformada de Fourier (FT-IR)}

En el Cuadro $\mathrm{N}^{\circ} 8$ se presentan las intensidades de absorbancia relativas de los números de onda asignados a los principales polímeros de la madera vía el análisis del material mediante FT-IR.

\section{Cuadro $\mathbf{N}^{\circ} 8$ \\ RELACIÓN OBTENIDA ENTRE LA INTENSIDAD DE ABSORBANCIA DEL NÚMERO DE ONDA DESIGNADO A LOS POLÍMEROS}

\begin{tabular}{|c|c|c|c|c|}
\hline \multirow{2}{*}{ Tratamiento* } & \multirow{2}{*}{$\begin{array}{l}\text { Condición } \\
\text { Muestra** }^{\star *}\end{array}$} & \multicolumn{3}{|c|}{ Intensidades Relativas ${ }^{\star \star \star}$} \\
\hline & & $I_{1508} / I_{1164}$ & $I_{1508} / I_{896}$ & $I_{1508} / I_{1734}$ \\
\hline \multirow{2}{*}{$\mathbf{T}$} & $\mathrm{t}$ & 0,56 & 1,61 & 3,04 \\
\hline & $\mathrm{tx}-\mathrm{pr}$ & $0,85-0,77$ & $4,41-4,25$ & $3,99-5,03$ \\
\hline \multirow{2}{*}{ CCA-C } & $\mathrm{t}$ & 0,6 & 2,44 & 2,44 \\
\hline & $\mathrm{tx}-\mathrm{pr}$ & $0,66-0,65$ & $2,6-3,43$ & $3,11-2,99$ \\
\hline \multirow{2}{*}{$\mathrm{BIO}$} & $\mathrm{t}$ & 0,52 & 2,63 & 2,25 \\
\hline & $\mathrm{tx}-\mathrm{pr}$ & $0,59-0,64$ & $2,33-2,68$ & $2,16-2,3$ \\
\hline \multirow{2}{*}{ B010 } & $\mathrm{t}$ & 0,58 & 1,28 & 3,91 \\
\hline & $\mathrm{tx}-\mathrm{pr}$ & $0,84-0,7$ & $3,5-342$ & $2,88-2,32$ \\
\hline \multirow{2}{*}{ FB } & $\mathrm{t}$ & 0,55 & 2,43 & 2,73 \\
\hline & $\mathrm{tx}-\mathrm{pr}$ & $0,54-0,61$ & $2,5-1,49$ & $2,94-2,92$ \\
\hline \multirow{2}{*}{ B015 } & $\mathrm{t}$ & 0,54 & 2,37 & 2,41 \\
\hline & $\mathrm{tx}-\mathrm{pr}$ & $0,58-0,7$ & $1,7-3,28$ & $4,18-3,54$ \\
\hline \multirow{2}{*}{ P25 } & $\mathrm{t}$ & 0,62 & 1,64 & 3,57 \\
\hline & $\mathrm{tx}-\mathrm{pr}$ & $0,9-0,83$ & $3,74-2,5$ & $3,29-3,78$ \\
\hline \multirow{2}{*}{ P50 } & $\mathrm{t}$ & 0,58 & 2,45 & 2,44 \\
\hline & $t x-p r$ & $0,96-1,05$ & $4,19-3,57$ & $4,08-2,72$ \\
\hline \multirow{2}{*}{ P100 } & $t$ & 0,55 & 2,37 & 2,47 \\
\hline & $\mathrm{tx}-\mathrm{pr}$ & $0,89-0,87$ & $2,49-5,63$ & $3,44-3,39$ \\
\hline
\end{tabular}

${ }^{*}$ CCA-C: Arseniato de cobre cromatado al $2 \%$;

BIO: preservante biodegradable al $5 \%$;

BO10: Metaborato de sodio al 10\%; FB: Fosfato borato al $8 \%$;

BO15: Metaborato de sodio al 15\%;

P: Paraformaldehído al $25 \%, 50 \%$ y $100 \%$.

**t: testigo de cada tratamiento, sin exposición a la cepa de pudrición castaña;

tx: exposición a G. sepiarium mediante ensayos de toxicidad;

pr: exposición a G. sepiarium mediante ensayos de permanencia *** 11508 / 11164: relación lignina-

holocelulosa; 11508 / 1896: relación lignina-celulosa; 11508 / I1734: relación lignina-hemicelulosas.

Diferentes reportes avalan la utilidad del análisis de las intensidades de absorbancia relativas (IARs) de los espectros FT-IR para la caracterización de materiales lignocelulósicos expuestos a degradacion fúngica (Moore y Owen, 2001; Pandey y Pitman, 2003). En correspondencia con esto, las IARs $I_{1508} / l_{1164}, I_{1508} / l_{896}$ e $I_{1508} / I_{1734}$ del material proveniente de ambos 
ensayos (tx y pr) fueron compatibles con la habilidad de G. sepiarium para degradar selectivamente los polisacáridos de la madera de pino ponderosa. Las mayores IARs o tendencia en este sentido en la madera expuesta a biodeterioro ( $\mathrm{t} v s \mathrm{tx} / \mathrm{t} v s \mathrm{pr}$ ) revelaron dicho patrón de despolimerización (Cuadro $\mathrm{N}^{\circ} 8$ ). Pandey y Pitman (2003) arribaron a iguales resultados con albura de Pinus sylvestris expuesta a Coniophora puteana, responsable de pudrición castaña: esta especie removió selectivamente los carbohidratos provocando un aumento en la relación lignina/carbohidratos con el avance de la degradacion. La madera bajo estudio también presentó una coloración castaña característica, compatible con la alteración oxidativa de la lignina que ocurre en este tipo de deterioro (Blanchette, 1995; Schwarze, 2007).

Asimismo, excepto para las muestras tratadas con los boratos, las IARs (principalmente $\mathrm{I}_{1508} / \mathrm{l}_{1164}$ ) se correspondieron con la toxicidad de los preservantes determinada indirectamente mediante porcentaje de pérdida de peso (Pp). En este sentido, los mayores valores obtenidos para los tratamientos P (P25, P50 y P100) fueron los esperados tomando en consideración la baja resistencia al deterioro (la menor respecto del resto de los tratamientos) que dichas soluciones le confirieron al material (Cuadro $\mathrm{N}^{\circ} 3$ ).

En cuanto a las muestras con CCA, la $\mathrm{I}_{1508} / \mathrm{l}_{1164}$ (valor vs su $\mathrm{t} \leq 0,10$ y uno de los menores obtenidos) se correspondió con la alta resistencia estimada para dicho material. Por su parte $\mathrm{I}_{1508} / \mathrm{l}_{896}$ e $\mathrm{I}_{1508} / \mathrm{I}_{1734}$ fueron mayores a t, indicando en consecuencia, degradación de la celulosa y hemicelulosas (estas últimas por sobre la celulosa).

Lo obtenido fue compatible con el patrón de degradación de polisacáridos que producen las especies responsables de este tipo de deterioro en las primeras etapas del proceso de degradación (Pp $\leq 10 \%$ : Winandy y Morrell, 1993; Curling et al., 2002) porcentaje de Pp estimado para la madera de este tratamiento (Cuadro $\mathrm{N}^{\circ} 3$ ).

En cuanto al material con BIO, $\mathrm{l}_{1508} / \mathrm{l}_{1164} \mathrm{e} \mathrm{I}_{1508} / \mathrm{l}_{1734}$ (pertenecientes al grupo de los valores más bajos obtenidos) tambien se correspondieron con la toxicidad determinada para esta solución (Cuadro $\mathrm{N}^{\circ} 3$ ); respecto de $\mathrm{I}_{1508} / \mathrm{I}_{896}$, lo hallado podría deberse al aumento relativo de la celulosa durante las primeras etapas del proceso de degradación, momento en el cual, según se indicó, las hemicelulosas son preferentemente atacadas. De acuerdo con esto, mediante FT-IR también quedó evidenciada la mayor toxicidad de la solución BIO (valores similares al de su correspondiente $t y$, salvo muy pocas excepciones, los más bajos respecto de los tratamientos con Boro y con $\mathrm{P}$ ).

En referencia a las muestras tratadas con $\mathrm{BO} 10, \mathrm{BO} 15$ y $\mathrm{FB}$, las IARs no se correspondieron con las Pp estimadas (toxicidad) (Cuadro $N^{\circ} 3$ ). La formación de complejos BoroBoro y/o Boro-compuestos orgánicos determinarían el solapamiento de picos de absorbancia y, a causa de ello, interferencias en los resultados (Peak et al., 2003).

Respecto de los ensayos de permanencia, en términos generales las IARs no se correspondieron con dicho comportamiento. De acuerdo con esto, en esta investigación, si bien las IARs permitieron identificar el curso y modo de degradación del material, solo fueron compatibles con la toxicidad (y consecuentemente con la pérdida de peso) de algunas de las soluciones. A causa de ello, las IARs no aportaron resultados concluyentes en torno a la toxicidad y permanencia diferencial entre las soluciones ensayadas; estas solo aportaron indicios en cuanto a la diferente toxicidad entre alguna de ellas. FT-IR no sería un indicador fidedigno del comportamiento de preservantes solubles que puedan tener reacciones que generen subproductos insolubles. Esto tal vez es consecuencia de interacciones en las muestras como resultado de la repolimerización y/o precipitación, involucrando los principios activos de los químicos, metabolitos fúngicos y/o productos de degradación en el material y la consecuente detección de niveles de absorbancia que no reflejan el contenido real de holocelulosa y lignina de la madera de partida. En este sentido, existen antecedentes acerca de la formación de complejos lignina-CCA ( $\mathrm{Cr}$ ) que alteran las lecturas FT-IR. Respecto de los compuestos en base a Boro, el pH, la estructura de la molécula y su polimerización (esto último estrechamente vinculado a su concentración), afectan los espectros infrarrojos (Moore y Owen, 2001; Peak et al., 2003).

En el Cuadro $N^{\circ} 9$ se presenta un ranking de productos resultante de la durabilidad por 
tipo de ensayo que cada uno de ellos confirió a la madera. Además se asocia la clase de resistencia natural y adquirida de la madera de pino ponderosa a las categorías de riesgo de uso según norma IRAM 9600 (1998) para las que la madera de los distintos tratamientos presentaría aptitud.

\section{Cuadro $\mathrm{N}^{\circ} 9$ \\ RANKING DE PRODUCTOS SEGÚN RESISTENCIA CONFERIDA A LA MADERA Y CLASE DE RIESGO/SITUACIONES DE USO POSIBLES PARA EL MATERIAL (IRAM 9600)}

\begin{tabular}{|c|c|c|c|c|}
\hline Ranking & Producto* & Toxicidad & Permanencia & Categoría de Riesgo \\
\hline \multicolumn{2}{|c|}{ CCA-C } & A. Resistente & A. Resistente & $\mathrm{R} 2 \mathrm{a}$ R6 \\
\hline 1 & $\mathrm{BIO}$ & A. Resistente & A. Resistente & R2 a R5 \\
\hline 2 & $\mathrm{BO} 10$ & A. Resistente & Resistente & $\mathrm{R}^{2} \mathrm{a} \mathrm{R}^{\mathrm{T}}$ \\
\hline 3 & FB & Resistente & Resistente & $\mathrm{R} 2 \mathrm{a} \mathrm{R}^{1}$ \\
\hline 4 & BO15 & Resistente & Resistente & $\mathrm{R}^{2} \mathrm{a} \mathrm{R}^{\mathrm{T}}$ \\
\hline 5 & P25 & M. Resistente & M. Resistente & $\mathrm{R}^{2}{ }^{2}$ \\
\hline 5 & P50 & M. Resistente & M. Resistente & $\mathrm{R}^{2}{ }^{2}$ \\
\hline 5 & P100 & M. Resistente & M. Resistente & $\mathrm{R} 2^{2}$ \\
\hline \multicolumn{2}{|c|}{ Testigo } & M. Resistente & M. Resistente & $\mathrm{R}^{2}{ }^{2}$ \\
\hline \multicolumn{5}{|c|}{$\begin{array}{l}\text { *Tratamientos: CCA-C: Arseniato de cobre cromatado al } 2 \% \text {; } \\
\text { BIO: preservante biodegradable al } 5 \% \text {; } \\
\text { BO10: Metaborato de sodio al } 10 \% \text {; } \\
\text { FB: Fosfato-borato al } 8 \% \text {; BO15: Metaborato de sodio al } 15 \% \text {; } \\
\text { P: Paraformaldehído al } 25 \%, 50 \% \text { y } 100 \% \text {. } \\
\text { 1: La protección por diseño o recubrimientos hidrófugos en } \mathrm{R} 3 \text { incrementaría su vida en servicio } \\
\text { particularmente como madera estructural. } \\
\text { 2: En esta situación con posibilidades de humectación ocasional }(\mathrm{CH}>20 \% \text { promueve el ataque } \\
\text { por hongos xilófagos) evitar su uso como madera estructural. }\end{array}$} \\
\hline
\end{tabular}

\section{CONCLUSIÓN}

La albura de pino ponderosa es moderadamente resistente al ataque de G. sepiarium, hongo con habilidad para causar pudrición castaña.

Si bien las soluciones en base a Boro y $\mathrm{BIO}$ otorgan a esta madera una resistencia mayor (durabilidad adquirida) a la que naturalmente posee, BIO poseería el mayor potencial como sustituto del CCA. La toxicidad de dicha solución evidenciada indirectamente mediante porcentaje de pérdida de peso y espectroscopía infrarroja transformada de Fourier (FT-IR) como también los parámetros de preservación obtenidos en el material, avalan su aptitud. Por su parte, si bien manifestó tendencia al lixiviado, el material mantuvo su condición de altamente resistente a la degradación fúngica.

Respecto del análisis de la madera mediante FT-IR, si bien las intensidades de absorbancia relativas permitieron evidenciar el curso y modo de degradación del material perteneciente a los 2 ensayos establecidos, estas solo fueron compatibles con la toxicidad de algunas de las soluciones. El solapamiento entre picos de absorbancia de los componentes 
estructurales de la madera y otros polímeros resultantes de la transformación y polimerización de los principios activos y su interacción puede limitar su uso como único estimador del comportamiento del producto usado.

\section{REFERENCIAS}

ASTM D-2017-81 (Reapproved 1986), 1986. Standard Method of Accelerated Laboratory Test of Natural Decay Resistance of Woods. ASTM Book of Standars, American National Standar, pag.: 348-352.

Bernardis, A. C. y Popoff, O., 2009. Durability of Pinus elliottii wood impregnated with Quebracho colorado (Schinopsis balansae) bio-protectives extracts and CCA. Maderas, Ciencia y Tecnología 11(2):107-115.

Blanchette, R., 1995. Degradation of the lignocellulose complex in wood. Canadian Journal of Botany 73(Suppl.1):999-1010.

Borrallo Jiménez, M., 2007. Construcción en madera: influencia de los protectores de madera basados en sales hidrosolubles en la corrosión de herrajes metálicos. Informes de la construcción 59: 43-49.

Bozo G. A; Silva, R. y Montero, D., 2010. Resistencia a la flexión estática de madera de Pinus radiata D. Don. impreganada con cobre alcalino cuaternario (ACQ-D). Actas II Congreso Iberoamericano de Protección de la Madera (A46), RIPMA, Mar del Plata, Argentina.

Caldeira, F., 2010. Boron in wood preservation. Review in its physico-chemical aspects. Silva Lusitana 18(2):179-196.

Carmona Cerdá, R. y Barahona Neyra, A., 2010. Desempeño de madera de Pinus radiata D. Don. impregnada con cobre alcalino cuaternario (ACQ-D) frente a hongos de pudrición y Termitas subterráneas. Actas II Congreso Iberoamericano de Protección de la Madera (A28), RIPMA, Mar del Plata, Argentina.

Carmona, R.; Avrella, E. C. y Elgueta, R., 2012. Boratos: Una alternativa al CCA para la protección de la madera de Pinus radiata D. Don contra termitas subterráneas en aplicaciones de bajo riesgo. Actas III Congreso Iberoamericano de Protección de la Madera, RIPMA, Concepción, Chile.

Coudert, L.; Blais, J. F.; Mercier, G.; Cooper, P; Janin, A. y Gastonguay, L., 2014. Demonstration of the efficiency and robustness of an acid leaching process to remove metals from various CCA-treated wood samples. Jour. Environm. Manag., 132:197-206.

Coudert, L.; Blais, J. F.; Mercier, G.; Cooper, P.; Gastonguay, L.; Morris, P.; Janin, A. y Reynier, N., 2013. Pilot-scale investigation of the robustness and efficiency of a copper-based treated wood wastes recycling process. Journal of Hazardous Materials 261:277-285.

Curling, S.; Clausen, C. A. y Winandy, J. E., 2002. Experimental method to quantify progressive stages of decay of wood by basidiomycetes fungi. International Biodeterioration \& Biodegradation 49:13-19.

Cvikrova, M.; Mala, J.; Hrubcova, M. and Eder, J.; 2006. Soluble and cell wall-bound phenolics and lignin in Ascocalyx abietina infected Norway spruces. Plant Science 170:563-570.

Díaz, B.; Murace, M.; Peri, P.; Keil, G.; Luna, M. L. y Otaño, M., 2003. Natural and preservative treated durability of Populus nigra cv Italica timber grown in Santa Cruz Province, Argentina. International Biodeterioration \& Biodegradation 52:43-47.

Encina, O., 2004. Conservación de maderas. Grupo de Investigación en Conservación de Maderas (GICOM), Universidad de Los Andes, Taller de Tratamiento de Maderas, Museo Salvador Valero, Núcleo Rafael Rangel Ula. $22 \mathrm{pp}$.

EPA, 2016. Overview of Wood Preservative Chemicals. Agencia de Protección Ambiental de EEUU.. www.epa.gov.

Escobar, E., 2012. Reemplazo de CCA para madera de construcción en Chile. III Congreso Iberoamericano de Protección de la Madera. Concepción, Chile.

Espiñeira, J. M.; Novo Uzal, E.; Gómez Ros, L. V.; Carrión, J. S.; Merino, F.; Ros Barceló, A. y Pomar, F., 2011. Distribution of lignin monomers and the evolution of lignification among lower plants. Plant Biology 13:5968. 
Gherscovic, D. A., 2012. La situación de la protección industrial de la madera en Argentina. III Congreso lberoamericano de Protección de la Madera. Concepción, Chile.

Girardo Ramírez, M., 2010. Evaluación preliminar de preservantes para el control de termitas de madera seca en cuatro maderas provenientes de plantaciones forestales. Actas II Congreso lberoamericano de Protección de la Madera (A358), RIPMA, Mar del Plata, Argentina.

Highley, T. L.; Clausen, C. A.; Croan, S. C.; Green, F.; Illman, B. L. y Micales, J. A., 1994. Research on biodeterioration of wood, 1987-1992. I. Decay mechanisms and biocontrol. USDA Forest Service, Research Paper FPL-RP-529.

Hinterstoisser, B.; Jalkanen, R.; Nowotny, M. y Schwanninger, M., 2001. Lignification of Scots Pine trees from Arctic Circle up to timberline. Buvísindi Icel. Agr. Sci., 14:55-59.

IRAM 9518, 1962. Toxicidad, Permanencia y Eficacia de Preservadores de Madera. Instituto Argentino de Racionalización de Materiales. 12 pp.

IRAM 9600, 1998. Preservación de maderas. Maderas preservadas mediante procesos con presión en autoclave. $23 \mathrm{pp}$.

Kose, C. y Kartal, S. N., 2010. Tolerance of brown-rot and dry-rot fungi to CCA and ACQ wood preservatives. Turk. J. Agric. For., 34:181-190.

Keil, G.; Maly, L.; De Cristófano, N.; Refort, M.; Acciaresi, G., 2012. Comportamiento a la impregnación por vacío y presión de las maderas de Pinus ponderosa Dougl. Ex Laws y Araucaria angustifolia (Bertol.) Kuntze. Revista Facultad de Agronomía 111(2):99-110.

Lebow, S. T., 2010. Wood preservation In: Wood Handbook - Wood as an engineering material: U.S. Department of Agriculture Forest Service. Forest Products Laboratory. General Technical Report FPL-GTR-190. Madison, Wisconsin, pag.: 1-15 a 1-28.

Luna, M. L.; Ramos Giacosa, J. P.; Giudice, G. E.; Fernández, P. V.; Ciancia, M. y Saparrat, M., 2015. Structure and chemistry of the xylem of arborescent species of Blechnum from South America. IAWA Journal $36: 3-21$.

Moore, A. K. y Owen, N. L., 2001. Infrared Spectroscopic Studies of Solid Wood. Applied. Spectroscopy Reviews $36(1): 65-86$.

Moreno, G.; Woottitz, A.; Carranza, M. E. y Segienowicz, R., 2010. Fijación del preservante CCA en maderas de Pinus sp y Eucalyptus grandis (Hill ex Maiden) y su influencia sobre el ambiente. Actas II Congreso Iberoamericano de Protección de la Madera (A15), RIPMA, Mar del Plata, Argentina.

Murace, M; Spavento, E.; Rivas, P.; Saparrat, M. y Keil G., 2014. Comportamiento de la madera del pino ponderosa (Pinus ponderosa Dougl. ex. Laws.) expuesta a degradación por Gloeophyllum sepiarium (Gloeophyllales, Basidiomycota). Quebracho- Revista de Ciencias Forestales 22(1,2):114-124.

Murgia, M., 2012. Desarrollo de nuevos productos para la preservación de maderas. Laboratorio de Química Aplicada. Facultad de Bioquímica y Ciencias Biológicas. Universidad Nacional del Litoral. Santa Fe. Argentina. 10 pp.

Paes, J. B.; Coutinho Ramos, I. E. y Barbosa do Nascimento, J. W., 2008. Eficiência do CCB na resistência da madeira de algaroba (Prosopis juliflora (Sw.) D.C.) a cupins xilófagos, em ensaio de alimentação forçada. Floresta e Ambiente 15(1):1-12.

Pandey, K. K. y Pitman, A. J., 2003. FTIR studies of the changes in wood chemistry following decay by brown-rot and white-rot fungi. International Biodeterioration \& Biodegradation 52:151-160.

Peak, D.; Luther, G. W. y Sparks, D. L., 2003. Atr-Ftir spectroscopic studies of boric acid adsorption on hydrous ferric oxide. Geochimica et Cosmochimica Acta 67(14):2551-2560.

Rodríguez Barreal, J. A., 1998. Problemática Ambiental. En: Patología de la Madera. Escuela Técnica Superior de Ingenieros de Montes. Madrid. $253 \mathrm{pp}$.

Saparrat, M. C.; Estevez, J. M.; Troncozo, M. I.; Arambarri, A. y Balatti, P., 2010. In-vitro depolymerization of Scutia buxifolia leaf-litter by a dominant Ascomycota Ciliochorella sp. Int. Biodeterior. Biodegrad., 64: 262-266. 
Schmidt, O., 2006. Wood and tree decay. Biology, damage, protection and use. Springer-Verlag Berlin Heidelberg, Germany. 251 pp.

Schwarze, F. W. M. R., 2007. Wood decay under microscope. Fungal Biology Reviews 21:133-170.

Thévenon, M. F.; Tondi, G. and Pizzi, A., 2010. Environmentally friendly wood preservative system base on polymerized tannnin-resin-boric acid for outdoor applications. Maderas, Ciencia y Tecnología 12(3): 253-257.

USDA, Forest Service, 2010. Wood Handbook. Wood as an engieneering material. Department of Agriculture. United States. $509 \mathrm{pp}$.

Walker, J. C. F., 2006. Primary wood processing. Principles and practice. 2da. Edición. Sringer. 556 pp.

Winandy, J. E. y Morrell, J. J., 1993. Relationship between incipient decay, strength, and chemical composition of Douglas-Fir heartwood. Wood and Fiber Science 25(3):278-288.

Wottitz, C. A. y Moreno, G. A., 2011. Fijación química del preservante CCA-C en la madera de Pinus elliottii. Parte 1: influencia de la temperatura y de la humedad relativa. Madera, Ciencia y Tecnología 13(1):85-103.

Wright, J. E. y Deschamps, J. R., 1976/77. Basidiomicetos xilófilos de la Región Mesopotámica. III. Los géneros Bjerkandera, Gloeophyllum, Gloeoporus, Hirschioporus, Hydnolyporus, Phaeocoriolellus, Pycnoporus y Xerotinus. Rev. Inv. Agrop. Serie 5, Patología vegetal 13(2): 27-52.

Zabel, R. A. y Morrell J. J., 1992. Wood microbiology. Decay and its prevention. Academics Press Inc., 476 pp. 
